Discussion Workplace culture was seen as a barrier to healthy eating. Initiatives designed to modify work culture may prove effective as a means by which to promote healthy eating in the organisational setting.

\section{THE ROLE OF AGE AND HEALTH IN RETURNING TO WORK: RESULTS FROM THE SUPPORTING OLDER PEOPLE INTO EMPLOYMENT (SOPIE) COHORT}

1J Brown*, ${ }^{2}$ SV Katikireddi, ${ }^{2}$ AH Leyland, ${ }^{3}$ RW McQuaid, ${ }^{4} \mathrm{~J}$ Frank, ${ }^{1}$ EB Macdonald. ${ }^{1}$ Healthy Working Lives Group, Institute of Health and Wellbeing, University of Glasgow; ${ }^{2} \mathrm{MRC} / \mathrm{CSO}$ Social and Public Health Sciences Unit, Institute of Health and Wellbeing, University of Glasgow; ${ }^{3}$ Stirling Management School, University of Stirling, Stirling; ${ }^{4}$ Scottish Collaboration for Public Health Research and Policy, University of Edinburgh

\subsection{6/oemed-2018-ICOHabstracts. 132}

Introduction By 2020 people aged 50 years and over will make up almost half of the adult population in the UK. Policy aims to enable more people to work for longer however there is a dramatic drop in labour participation after age 50. Our aim was to investigate the impact of age, and health on return to work (RTW) in welfare benefit claimants engaging with the Work Programme (WP); the UK Government's main RTW initiative. It supports two main groups of claimants for two years - Job Seeker Allowance (JSA), for people who are unemployed but capable of work; Employment Support Allowance (ESA), for people with a disability that makes it more difficult to work.

Methods The data were from the SOPIE cohort (13 461 unemployed clients aged 18-64, who entered the WP in Scotland in 2013/2014). Data were analysed using STATA 14 and a Poisson modelling approach using fractional polynomials to model age as a continuous variable.

Results Clients aged 50 and over accounted for 15\% of JSA and $30 \%$ ESA groups. The proportion of clients disclosing health conditions (HC) were: 'JSA under-50', 25\%; 'JSA over50', 53\%; 'ESA under-50', 97\%; 'ESA over-50', 98\%. Multiple $\mathrm{HC}$ were more common in ESA clients. Job start rates for clients were: 'JSA under-50', 65\%; 'JSA over-50', 49\%; 'ESA under-50', 23\%; 'ESA over-50', 14\%. There was a strong relationship between age, health and job start with the predicted probability of job start highest in the first three months of the WP. The analyses also investigated the influence of biopsychosocial factors on RTW.

Conclusion This study is on-going and will inform interventions focussing on addressing age-specific, health and biopsychosocial barriers for future RTW programmes with the aim of improving employment outcomes, so that not only individuals but employers and the economy can benefit from extending working lives.

\section{PHYSICAL REHABILITATION APPROACH FOR RETAINING HEALTH CARE WORKERS SUFFERING FROM MUSCULOSKELETAL DISORDERS} ${ }^{1}$ School of Specialisation in Occupational Health, University of Milan, Milan, Italy; ${ }^{2}$ Occupational Health Unit, San Gerardo Hospital, Monza, Italy; ${ }^{3}$ School of Medicine and Surgery, University of Milan Bicocca, Milan, Italy

\subsection{6/oemed-2018-ICOHabstracts. 133}

Introduction Healthcare professionals are known to be at high risk for work-related musculoskeletal disorders (MSDs). Physical rehabilitation may be an important approach for retaining
Health Care Workers (HCWs) suffering from these disorders, especially among older workers. The aim of this study was to evaluate the results of a rehabilitation program dedicated to the employees of a large hospital in the Northern Italy.

Methods HCWs with shoulder disorders (SD) and Low Back Pain (LBP) were identified by the Occupational Health Unit and admitted to a physical rehabilitation program. Functional assessment scales were administered at the beginning and the end of the program: UCLA and Constant scale for SD and Borg scale for LBP. We applied Wilcoxon test for statistical comparisons. The level of significance adopted was 5\%.

Results During a 24 months period, occupational physicians identified $123 \mathrm{HCWs}$ with MSDs. Workers were mainly nurses (37.5\%) and nursing assistive personnel (34.8\%), with an average length of service of $28.53 \pm 8.92$ years. Only 47 workers completed the rehabilitation program. The treated workers were mainly affected by LBP $(n=22,46.81 \%)$ and SD $(n=20,42.52 \%)$. After rehabilitation, significant improvements in the UCLA scale ( $p$-value: 0.0206) were observed among the subjects affected by SD. The improvements in the Borg scale were also significant (p-value: 0.0011) among the subjects affected by LBP. Occupational physicians prescribed work restrictions in only five subjects $(5.88 \%)$. Three workers with previous work restrictions were considered fully fit-forwork after treatment. The remaining subjects returned to work without any restrictions.

Conclusions A rehabilitation program appears to be a valuable approach for retaining older HCWs affected by MSDs. In our study, more than $88 \%$ of workers have positively evaluated the program. Occupational physicians may play an important role in this program, especially in the assessment of subjects with work-related problems.

\section{IMPACT OF INDIVIDUAL MOTIVATION DIFFERENCES ON REDUCING SEDENTARY BEHAVIOUR}

1,2 V Hermans* ${ }^{*}{ }^{2} \mathrm{~L}$ Van Naemen, ${ }^{3} \mathrm{~J}$ Seghers, $1,{ }^{4} \mathrm{~L}$ Godderis, 1,5 L Daenen. ${ }^{1} K$ nowledge, Information and Research Center, IDEWE Group (External Service for Prevention and Protection at Work), Leuven, Belgium; '2Department of Experimental and Applied Psychology, Work and Organisational Psychology (WOPS), Faculty of Psychology and Education Sciences Vrije Universiteit Brussel, Brussel, Belgium; ${ }^{3}$ Department of Kinesiology, Physical Activity, Sports \& Health Research Group, KU Leuven, Leuven, Belgium; ${ }^{4}$ Environment and Health, Faculty of Medicine, KU Leuven, Louvain, Belgium; ${ }^{5}$ Department of Rehabilitation Sciences and Physiotherapy, Human Physiology and Anatomy (KIMA), Faculty of Physical Education and Physiotherapy, Vrije Universiteit Brussel, Brussels, Belgium

\subsection{6/oemed-2018-ICOHabstracts.134}

Introduction Reviews small effects for interventions sitting behaviour. This study aimed at evaluating basic psychological needs (need for competence, for autonomy and for relatedness) reduction in sitting behaviour.

Methods This study is part of the Move@TheOffice RCTstudy in the offices a large pharmaceutical company. The experimental group (19 participants) received a multiintervention to reduce sitting. To measure the basic psychological needs, the Work-related Basic eed atisfaction cale was used. The BREQ-3 measured the degree of motivation regulation to reduce sitting. Sitting was measured using the micro Activ$\mathrm{Pal}^{\mathrm{TM}}$ monitor. Data were analysed using SPSS.

Results Significant decrease $(p<0,05)$ in sitting time was found the experimental group. A significant BREQ-index was found after the intervention $(p<0.05)$, a higher to decrease sitting 